\title{
1405 Mechanical Properties of Single- and Double-Walled Carbon Nanotubes under Hydrostatic Pressure
}

\author{
Toshiaki Natsuki, Shinshu University, 4-17-1 Wakasato, Nagano 380-8553 \\ Morinobu Endo, Shinshu University, 4-17-1 Wakasato, Nagano 380-8553 \\ Kiyoshi Kemmochi, Shinshu University, 3-15-1 Tokida, Ueda 386-8576
}

\begin{abstract}
A frame structural model is presented to study the elastic properties of carbon nanotubes (CNTs) under hydrostatic pressure. Simple formulae are given for the bulk modulus of CNTs under hydrostatic pressure as a function of chiral structures. The double-walled nanotubes are coupled together through the van der Waals force between the inner and outer nanotubes. According to this model, the effects of the CNT structures with the armchair and zigzag on the pressure behavior are investigated based on closed-form elastic solution. The computational result indicates that the bulk modulus is less sensitive to the nanotube structures except for very small tube diameter.
\end{abstract}

Keywords: Carbon nanotubes; Mechanical properties; Bulk behavior; Simulation

\section{Introduction}

Carbon nanotubes (CNTs) have been the subject of intense research due to their exceptional mechanical, thermal and electrical properties. Experiment methods for measuring the mechanical properties are based mainly on the techniques of transmission electron microscopy (TEM) and atomic force microscopy (AFM) [1,2]. These studies have contributed to confirming that CNTs have exceptional mechanical properties. Although many theortical and experimental methods have been presented to estimate and measure the mechanical behaviors such as tensile stiffness and strength of CNTs, to the author' knowledge, few studies have been reported on the elastic response of an individual CNT to hydrostatic pressure. In this work, we present a theoretical study on the high pressure behavior of CNTs based on the solid mechanics with elastic continuum model. According to the present model, closed-form elastic solutions are obtained to predict the mechanical properties of single- and double-walled carbon nanotubes under hydrostatic pressure. Their mechanical properties, such as the strain and buck modulus of CNT structures with zigzag and armchair are discussed as a function of nanotube diameters.

\section{Theoretical approach}

The molecular structural mechanics approach based on a link between molecular and solid mechanics is applied to analyze the mechanical properties of CNTs under hydrostatic pressure. The atoms in the nanotube structures are considered as individual bodies connected with bond energy. Double-walled carbon nanotubes (DWNT) can be regarded as two nested single-walled carbon nanotubes (SWNTs). The DWNTs are coupled together through the van der Waals force (vdW) between the inner and outer nanotubes. The $\mathrm{vdW}$ interaction energy potential, as a function of the interlayer spacing between the inner and outer nanotubes, can be estimated by the Lennard-Jones potential. The interaction pressure between two adjacent nanotubes is assumed to be linear with the deflections in the radial direction of CNTs.

\section{Results and Discussion}

Many studies have been shown that the CNTs are subjected to an elastic response under a hydrostatic pressure of less than $2 \mathrm{GPa}$. In the present study, we take the hydrostatic pressure of $1.0 \mathrm{GPa}$. Figure 1 shows the results of the radial strain of capped CNTs as a function of diameters for zigzag and armchair nanotubes. The radial displacement exhibits a linear function of tube diameters and is insensitive to the structures of zigzag and armchair tubes. This result is very similar to the approach proposed by Li and Chou [3]. The bulking modulus of SCNT versus diameter for zigzag and armehair tubes is shown in Fig. 2. It is observed that the bulking modulus decreases with increasing nanotube diameter. This calculation predicts structural dependence of the bulk modulus since SWNTs are a range of $0.7 \sim 2.5 \mathrm{~nm}$ in diameters. According to calculated data plotted in Fig. 2, we can estimate the bulking modulus in typical nanotubes as $0.2 \mathrm{TPa}$, which are excellent agreement with the result obtained by Reich et al. [4]. For very small nanotube diameter (less than $0.8 \mathrm{~nm}$ ), the armchair tubes have larger bulk modulus than the zigzag tubes. Figure 3 shows the results of the radial strain of nanotubes as a function of the outer tube diameters. The simulated result of DWNT is a nonlinear dependence on the nanotube diameter, which is dissimilar to that of SWNT. For narrow CNTs with identical outer diameter, the outer tube of DWNTs has almost the same axial strain as SWNTs. This suggests that there exists samll vdW interaction between the inner and outer tubes under the hydrostatic pressure. As the nanotube diameter is increased, the outer tube of DWNTs has low strain than the SWNTs because the inner outer has stronger action against the outer tube by the vdW interaction. The inner of the DWNTs tends to the same strain as its outer tube with increasing nanotube diameter. Figure 4 shows the 
bulk modulus of both SWNTs and DWNTs versus nanotube diameter under the hydrostatic pressure. For the DWNTs, the interlayer spacings of $0.34 \mathrm{~nm}$ and $0.38 \mathrm{~nm}$ are taken to investigate the bulk behaviors. It is seen that the bulk modulus for the DWNT with $0.34 \mathrm{~nm}$ spacing are larger than that with larger interlayer spacing $(0.38 \mathrm{~nm})$, and the SWNT has the smallest bulk modulus in the identical diameter.

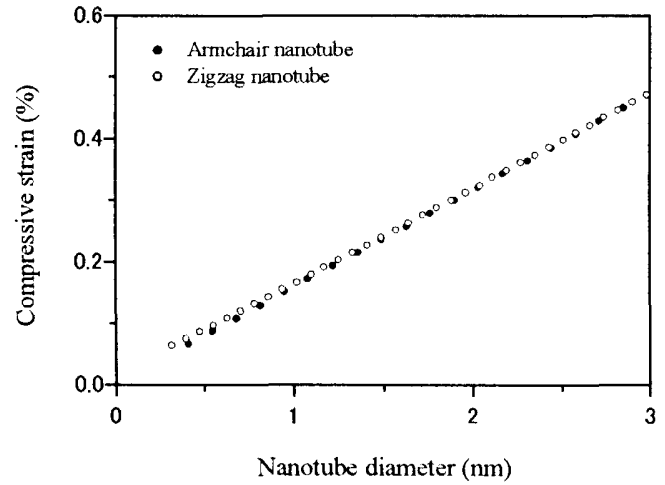

Fig 1 Variation of the compressive strain with the nanotube diameter

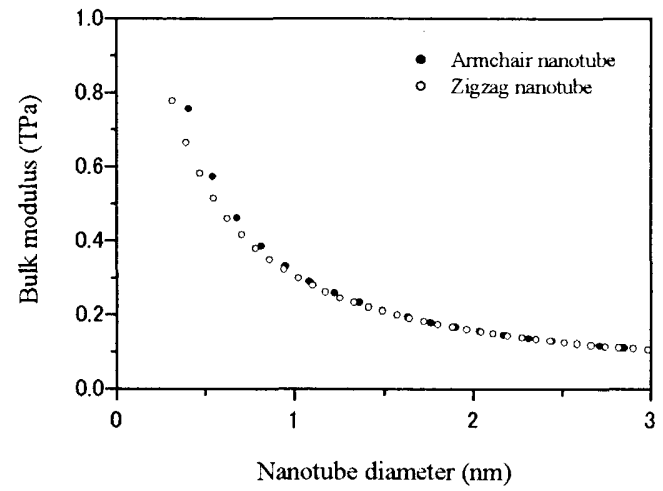

Fig. 2 Variation of the bulk modulus with the nanotube diameter

\section{Results and Discussion}

Using elastic continuum analysis of atomistic-based constitutive model, we obtain simple formulae to calculate the mechanical properties of not only SWNTs but also DWNTs under hydrostatic pressure. The result indicates that the bulk modulus is less sensitive to the nanotube structures except for very small tube diameter. The influence of the cap on the bulking modulus of the zigzag nanotubes is larger than that of the armchair nanotubes. For DWNTs, the spacing layer distance between the inner and outer nanotubes has lager influence on the bulking modulus and the strain.

\section{Acknowledgements}

This work was supported by the CLUSTER of Ministry of Education, Culture, Sports, Science and Technology, Japan.

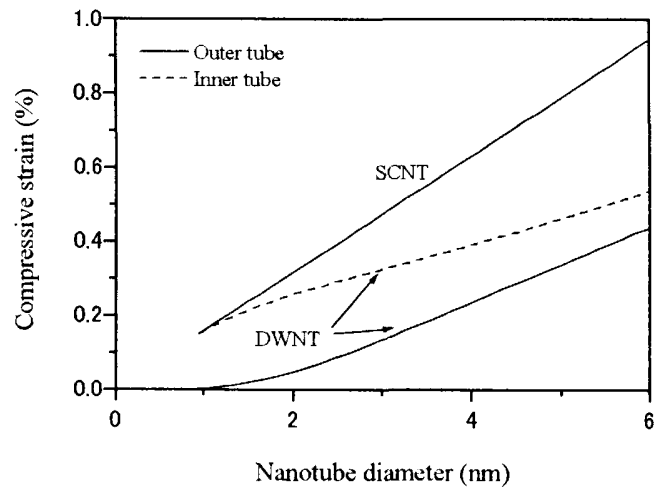

Fig. 3 The compressive strains in single- and doublewalled CNTs as a function of tube diameter

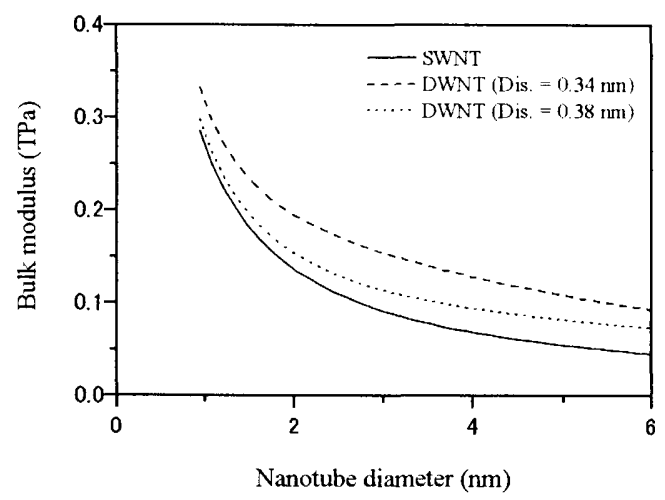

Fig. 4 The Bulk modulus in single- and double-walled CNTs as a function of tube diameter: The diameter of DWNT refers to the outer tube

\section{References}

[1] Treacy MMJ, Ebbesen TW, Gibson JM. Exceptionally high young's modulus observed for individual nanotubes Nature 1996;381:678-680

[2] Wong EW, Sheehan PE, Lieber CM. Nanobeam mechanics: Elasticity, strength, and toughness of nanorods and nanotubes. Scince 1997;277:1971-1975.

[3] Li C, Chou TW, Elastic properties of single-walled carbon nanotubes in transverse directions. Phys. Rev. B 2004:69:073401-1-3

[4] Reich S, Thomsen C, Ordejon P, Elastic properties of carbon nanotubes under hydrostatic pressure. Phys. Rev. B $202 ; 65 ; 153407-1-4$. 